\title{
A Bank Credit Model with Capital-Constrained Newsvendor Under Two Ordering Opportunities
}

\author{
Deli ZHAO \\ School of Management, University of Science and Technology of China, Hefei 230026, China \\ E-mail: zhaodeli@mail.ustc.edu.cn \\ Baofeng ZHANG \\ School of Management, University of Science and Technology of China, Hefei 230026, China \\ E-mail: zhangbf@mail.ustc.edu.cn \\ Zongshui WANG \\ School of Economics and Management, University of Chinese Academy of Sciences, Beijing 100190, \\ China \\ E-mail: wangzongshui8@163.com
}

\begin{abstract}
This paper proposes a financing system consisting of a bank under Mean-Variance criterion and a capital-constrained retailer, where the bank offers an unlimited credit to the retailer. The demand is assumed to be stochastic. The newsvendor is allowed to make an emergency order with a minimum reorder quantity threshold (RQT). It shows that under RQT, the newsvendor has different reorder strategies. The optimal primary order quantity and interest rate are derived, sequentially. Extension under perfectly competitive capital market is given. The mathematic model reveals that RQT and reorder price have significant effect on the optimal strategies.
\end{abstract}

Keywords capital constraint; two ordering opportunities; bank credit; newsvendor

\section{Introduction}

It is reported that small and medium-sized enterprises (SMEs) account for the majority of firms in the economy. To put it differently, SMEs play an important role in the economy and employment. However, a prevalent problem for SMEs or start-ups is the limited working capital, which has important impact on the optimal operational decisions and efficiency, such as manufacturing, ordering and advertising ${ }^{[1,2]}$, which, conversely, affects the economy performance, such as investment, employment, consumer welfare ${ }^{[3]}$. Therefore, how to solve this problem has been an important topic, which attracts great attention from the managers, scholars and policy-makers. Bank financing has been one of the most popular financing sources, which has been well analyzed form the financial perspective. However, the perspective of operationsfinance interfaces should be given more attention. To bridge this gap, this paper focuses on interfaces between the operations and finance, and derives Stackelberg equilibrium of bank

Received November 4, 2015, accepted January 7, 2016 
credit between the capital-constrained newsvendor and the bank and analyzes how the capital constraint impact the newsvendor's order decisions with two ordering opportunities.

This paper incorporates the two ordering opportunities with a minimum reorder quantity threshold into the capital constrained newsvendor under bank credit and focuses on the operations \& financing equilibrium. Due to the rapid progress of technology, the market competition intensifies, high product variety, the demand uncertainty of seasonal product is higher and higher. Retailers, especially for those with capital constraint, cannot obtain accurate market information for limited investment in information forecasting ${ }^{[4]}$. To solve this problem, many suppliers allow the retailers to order twice or more, that is, the retailer makes ordering decision before the sales season and then reorders when coming to or even during the sales season ${ }^{[5,6]}$. It shows that the double order policy benefits for the supply chain. However, an important question with double order to be considered is the setup cost allocation. Specifically, the supplier re-setups when the retailer reorders, which results extra costs. To reflect the sharing of the reset up cost, this paper assumes that there exists a minimum reorder quantity set by the supplier, that is, only when the reorder quantity is higher or equal to the RQT, the reorder policy is conducted. It is intuitive that higher reorder quantity threshold results in higher revenue for the supplier. One can conclude that the revenue from this threshold can at least covers the re-setup cost. On the other hand, this well-designed contract can be easily conducted in practice.

In this paper, we mainly focus on the interfaces between operations and finance, specifically, (i) the optimal ordering and credit pricing decisions; (ii) the effect of parameters, such as reorder quantity threshold, the reorder price on the optimal decisions. In order to explore the aforementioned questions, this paper considers a Stackelberg game between a deep-pocket bank and a capital-constrained newsvendor-like retailer, where the bank acts as the leader determining the optimal interest rate and the retailer as a follower determines the optimal order quantity. The retailer is allowed to order a single perishable good twice with a reorder quantity threshold and resells to the random market at fixed retail price in a single period. Stackelberg equilibrium is derived. The analytical results show that the reorder quantity threshold has a profound effect on the optimal results.

Existing literatures highly related to this research are mainly those considering problem under capital-constraint and credit financing. $\mathrm{Xu}$ and Birge ${ }^{[7]}$ among the first to explore the impact of capital constraints and capital structure on the firm's inventory decisions. Chao, et al. ${ }^{[8]}$ characterized the dependence of the firm's inventory strategy on the financial status in a dynamic framework. In a short-term bank credit area, Buzacott and Zhang ${ }^{[9]}$ focused on the asset-based financing problem with a capital-constrained retailer and a bank. Jing, et al. ${ }^{[10]}$ showed that which credit among bank credit and trade credit is more attractive depends on the production cost. Zhang, et al. ${ }^{[11]}$ incorporated the default risk into the financing problem and showed that the default risk has a significant impact on the decisions. Zhang and Zhao ${ }^{[12]}$ analyzed the decision of loan-to-value ratio of inventory financing under endogenous default risk and stochastic demand. $\mathrm{Ma}^{[13]}$ explored the impact of inventory financing, and trade credit, on the order strategy of retailer, separately. Another existing work is deserves our special attention. Dada and $\mathrm{Hu}^{[14]}$ studied how the bank finances the newsvendor, and proposed a 
non-linear loan schedule to coordinate the channel. Differently, we extend their basic model by incorporating the two order opportunities with RQT into the newsvendor's purchasing policy, and derive more general results.

The remainder of this paper is as follows. Basic model and notations are presented in Section 2. Equilibrium and analysis are derived in Section 3. The last section gives concluding remarks. Proofs of the propositions and corollaries are presented in the Appendix.

\section{Model Formulation and Notations}

Consider a Stackelberg game between a capital-constrained retailer (he) with initial capital $B$, and a bank (she). The bank offers unlimited credit to the retailer with interest rate $r$. The retailer is allowed to order a single good twice and sells to the stochastic market at a fixed retail price $p$. The retailer makes an primary ordering decision at fixed cost $w$ ahead of the sales season (time 0) with his initial capital plus the loan; and then reorders at the cost $e$ if the realized demand is high enough (time 1). At time 0 , the newsvendor can get a full information of the demand distribution, i.e., the cumulative density function (CDF) $F(x)$ and the probability density function (PDF) $f(x)$ on the interval $[0, b)$, where $b>0$. For simplicity, we assume that the salvage value of each unsold product to be zero and there is no goodwill loss for unmet demand. In fact, salvage value can be treated as a secured repayment, which results in a borrowing without bankruptcy case $\mathrm{e}^{[9]}$.

At time 1, the retailer can make an order if necessary. Taking the interests of supplier into account, it is assumed that the second order quantity is no smaller than $M$, that is, the supplier requires the retailer order at least $M$ at time 1. It is assumed that the retailer can borrow from the bank if he needs. Therefore, the repayment of loan for the primary order is uncertain due to the demand uncertainty. While the repayment of loan for the re-order is deterministic. Accordingly, we assume that the interest rate for reorder is the risk-free interest rate, $r_{f}$. Without loss of generality, we assume that $r_{f}=0$.

Some key assumptions are as follows and Table 1 collects the key notations.

Assumption 1 The failure rate of distribution $h(x)=\frac{f(x)}{1-F(x)}$ is increasing in $x$, that is, the demand distribution has an increasing failure rate (IFR) (see, e.g., [15]).

This assumption is imposed to guarantee the existence and uniqueness of solution to the optimization problem.

Assumption 2 To avoid trivial cases, we have $p>e>w>c$ and $p>w(1+r)$.

This assumption ensures each player earns a non-negative expected profit and joins the game.

Assumption 3 Perfect and complete information, that is, all the information about the agents and market is completely known to the agents.

Assumption 4 The capital market is assumed to be perfect (no tax, transaction costs and bankruptcy costs). 
Table 1 Key notations

\begin{tabular}{ll}
\hline Notation & Definition \\
\hline$p$ & Retail price \\
$w$ & Wholesale price \\
$e$ & Reorder price \\
$B$ & Initial capital \\
$r$ & Interest rate \\
$q$ & Primary order quantity \\
$x$ & Stochastic demand \\
$\pi$ & Profit of retailer/bank \\
\hline
\end{tabular}

\section{Equilibrium and Analysis}

In this section, backward induction is adopted to solve the two-level optimization problem. First, we analyze the retailer's optimal ordering strategy as a function of the interest rate set by the bank. Taking the retailer's best response and the initial capital into the bank's optimization problem, we compute the bank's strategy.

\subsection{The Retailer}

Before analyzing the newsvendor's problem with capital, we firstly give a benchmark case, where the newsvendor has a deep pocket. In this case, there exists only one restriction, i.e., minimum reorder quantity threshold (RQT). Only when the realized demand is higher than the primary order quantity, the retailer has a motivation to make a reorder decision. Specifically, if $x>q$, there would be a storage of $x-q$. More detail, if $x>q+M$, the retailer will reorder $x-q$. When $q<x<q+M$, the retailer reorders $M$ units if he chooses to reorder, and the corresponding profit is $p(x-q)-e M$. Thus, there is a demand threshold that the retailer is indifferent in reorder and non-reorder, i.e.,

$$
p(x-q)=e M .
$$

From (1), we can get the indifferent reorder point, i.e., $x_{2}(q)=\frac{e M}{p}+q$. It is straightforward that the reorder point is increasing in $M$, which suggests that $M$ has a significant impact on the reorder and primary order strategy. Therefore, due to the existence of the reorder quantity threshold, the retailer has three choices due to the demand realization and primary order quantity,

$$
Q=\left\{\begin{array}{lll}
0, & \text { if } & x<x_{2}, \\
M, & \text { if } & x_{2} \leq x<q+M, \\
x-q, & \text { if } & x \geq q+M .
\end{array}\right.
$$

Accordingly, the retailer's profit is given as

$$
\pi_{R}= \begin{cases}p x-w q, & \text { if } \quad x_{1} \leq x<q, \\ p q-w q, & \text { if } \quad q \leq x<x_{2}, \\ p x-w q-e M, & \text { if } \quad x_{2} \leq x<q+M, \\ (p-e) x-w q+e q, & \text { if } \quad x \geq q+M .\end{cases}
$$


Further, the retailer's expected profit is calculated as

$$
E \pi_{R}=(p-e) b+e q-w q-p \int_{0}^{q} F(x) \mathrm{d} x-p \int_{x_{2}}^{q+M} F(x) \mathrm{d} x-(p-e) \int_{q+M}^{b} F(x) \mathrm{d} x .
$$

Maximizing the expected profit of the retailer, we have the following theorem to show the optimal order quantity.

Theorem 1 The optimal primary order quantity with two ordering opportunities is determined by the following equation

$$
e \bar{F}(q+M)+p\left(\bar{F}(q)-\bar{F}\left(x_{2}\right)\right)-w=0
$$

In the following context, we mainly focus on the problem with capital constraint. Therefore, there exists two restrictions, i.e., the initial budget $B$, and the re-order quantity threshold $M$. To obtain the profit of the retailer, we firstly partition the demand. More specifically, if the $x<q$, the retailer would not make re-order decision. Further, if the realized demand is sufficiently low, that is, the sales revenue cannot cover the total debt $(w q-B)(1+r)$, the retailer goes bankrupt and bank only gets part of the loan, i.e., the sales revenue. Thus, there is a bankruptcy threshold, i.e., $x_{1}(q)=\frac{(w q-B)(1+r)}{p}$. It is intuitive that only when the demand realization is high enough that the retailer makes a reorder decision, which is the same as the above analysis. It is straightforward to verify that $0<x_{1}<q<x_{2}<q+M$.

Summarizing the above analysis, we get the profit trajectory of the retailer in each region, i.e.,

$$
\pi_{R}= \begin{cases}-B, & \text { if } \quad x<x_{1} \\ p x-B-(w q-B)(1+r), & \text { if } \quad x_{1} \leq x<q, \\ p q-B-(w q-B)(1+r), & \text { if } \quad q \leq x<x_{2}, \\ p x-B-(w q-B)(1+r)-e M, & \text { if } \quad x_{2} \leq x<q+M, \\ (p-e) x-B-(w q-B)(1+r)+e q, & \text { if } \quad x \geq q+M .\end{cases}
$$

Accordingly, the retailer's expected profit under bank credit is calculated given as

$$
\begin{aligned}
E \pi_{R}= & (p-e) b+e q-B-(w q-B)(1+r)-p \int_{x_{1}}^{q} F(x) \mathrm{d} x \\
& -p \int_{x_{2}}^{q+M} F(x) \mathrm{d} x-(p-e) \int_{q+M}^{b} F(x) \mathrm{d} x .
\end{aligned}
$$

Maximizing the retailer's expected profit, we have the Theorem 2, which reveals the optimal primary order quantity.

Theorem 2 For given the wholesale price, the reorder price and the initial capital, the optimal primary order quantity $q^{*}$ satisfies the following equation

$$
e \bar{F}\left(q^{*}+M\right)+p\left(\bar{F}\left(q^{*}\right)-\bar{F}\left(x_{2}\right)\right)-w(1+r) \bar{F}\left(x_{1}\right)=0 .
$$

This theorem shows that the condition that optimal primary order quantity satisfies is more complicated than that in classic newsvendor, which depends on the retailer's situation, the strategies given by the supplier, and that of the bank. It is intuitive that the primary order quantity is a trade-off between the wholesale price, retail price, reorder price, financing cost and random demand. 
To examine how these parameters affect the retailer's optimal ordering strategy, we give the following corollaries. Corollaries 1, 2 and 3 demonstrate the effect of the supplier's strategy on the optimal ordering quantity.

Corollary 1 The optimal primary order quantity is decreasing in the wholesale price.

It is intuitive that the wholesale price has a negative effect of the optimal decision.

Corollary 2 The optimal primary order quantity is increasing in the re-order price.

Corollary 2 shows that a higher reorder price results in a higher optimal order quantity. It is intuitive that, when $e \rightarrow p$, the optimal primary order quantity is raised to that without two order opportunities.

Corollary 3 The optimal primary order quantity is increasing in the reorder quantity threshold.

This corollary shows that the reorder quantity threshold has a significant effect on the optimal decision. To focus on its specific impact on the operational strategy, we give the following proposition.

Proposition 1 Given wholesale price, reorder price and initial capital:

(i) When $M$ is extremely low, i.e., $M=0$, the optimal primary order quantity $q_{0}^{*}$ is given as

$$
e \bar{F}\left(q_{0}^{*}\right)=w(1+r) \bar{F}\left(x_{1}\right),
$$

which is independent of the retail price.

(ii) When $M$ is extremely high, i.e., $M \rightarrow b$ the optimal primary order quantity $q_{m}^{*}$ is determined by

$$
p \bar{F}\left(q_{m}^{*}\right)=w(1+r) \bar{F}\left(x_{1}\right)
$$

(iii) $q_{0}^{*}<q_{m}^{*}$.

It is easy to verify the (i) and (ii) by letting $M=0$ and $M \rightarrow+\infty$ in Equation (7). Based on Corollary 3 , it is straightforward to get $q_{0}^{*}<q_{m}^{*}$. The second part is consistent with the result in [14].

From Proposition $1, q_{m}^{*}$ can be regarded as the optimal primary ordering level in the case in which the retailer can only make one order decision. Without the restriction of reorder quantity threshold the retailer's optimal primary order quantity equals to $q_{0}^{*}$. Therefore, we can conclude that when the supplier encourages the retailer to make re-order, the retailer will prefer to decrease the order number in the first-order stage and order more in the secondorder stage, and vice versa. It is intuitive to get that the reorder policy reduces the retailer's bankruptcy risk by reducing the primary order quantity. Further, RQT has a negative effect on the retailer's bankruptcy, that is, a bankruptcy-averse retailer prefers such operation strategy.

To analyze how the bank's strategy influences the optimal primary order quantity of the retailer, Corollary 4 is given.

Corollary 4 The optimal order quantity is decreasing in the interest rate.

A higher interest rate suggests a higher financial cost, which has a negative effect on the optimal decision. This corollary shows that the financial choice has a significant impact on the operational decision, which suggests that the interaction of the operation and finance should be paid more attention. 


\subsection{The Bank's Problem}

In this subsection, we proceed to focus on the risk-averse bank's problem on interest rate. It is assumed that the bank is deep pocket and carries on two businesses, i.e., financing the retailer and investing in the risk-free project. In this study, we assume that the bank charges two interest rates, which mainly depend on retailer's default risk. When the retailer chooses to reorder and borrows from the bank, there is no uncertainty, and consequently, the interest rate is the risk-free rate, which equals to investing the risk-free project. Therefore, the bank's profit financing the retailer equals the profit financing the retailer's primary order quantity, since the bank obtain zero profit form financing the reorder quantity. Thus, the profit of the bank is given as

$$
\pi_{b}=\left\{\begin{array}{lll}
p x-(w q-B), & \text { if } & x<x_{1}, \\
(w q-B) r, & \text { if } & x \geq x_{1} .
\end{array}\right.
$$

If the demand realization is relatively low, $x<x_{1}$, the retailer goes bankrupt with default and the bank only gets part of the loan. Therefore, the bank's expected total profit is

$$
E \pi_{b}=(w q-B) r-p \int_{0}^{x_{1}} F(x) \mathrm{d} x .
$$

The bank is assumed to be risk averse, measured with Mean-Variance ${ }^{[16]}$. Accordingly, the bank's utility function is given as

$$
U=E\left[\pi_{b}\right]-\lambda \operatorname{Var}\left[\pi_{b}\right],
$$

where $\pi_{b}$ is given as (10). Therefore, the variance of the bank's profit is calculated as

$$
\begin{aligned}
\operatorname{Var}\left[\pi_{b}\right] & =E\left[\pi_{b}^{2}\right]-E\left[\pi_{b}\right]^{2} \\
& =(w q-B)^{2} r^{2}-2 p \int_{0}^{x_{1}} x F(x) \mathrm{d} x+2 p^{2} x_{1} \int_{0}^{x_{1}} F(x) \mathrm{d} x-\left(p \int_{0}^{x_{1}} x F(x) d x\right)^{2} .
\end{aligned}
$$

Optimizing the risk-averse bank's problem under MV criterion, we get the following theorem to show the optimal interest rate.

Theorem 3 The optimal interest rate $r^{*}$ determined by the risk-averse bank under $M V$ criterion satisfies the following equation

$$
\begin{array}{r}
(w q-B) \bar{F}\left(x_{1}\right)-\lambda\left(2(w q-B) r^{*}+2 p \int_{0}^{x_{1}} F(x) d x-N F\left(x_{1}\right)\right) \\
-\left(w r^{*}\left(1-2 \lambda(w q-B) r^{*}\right)-w\left(1+r^{*}\right) F\left(x_{1}\right)(1-\lambda N)\right) T=0,
\end{array}
$$

where $N=(2 p-1) x_{1}+2 p\left(1-\int_{0}^{x_{1}} F(x) \mathrm{d} x\right)$ and $T=\frac{w F\left(x_{1}\right)\left(1-H\left(x_{1}\right)\right)}{K}$.

Equation (13) show that the FOC of the interest rate is much more complicated than that in [14] due to the following reasons, (i) the bank is risk averse under MV criterion; (ii) the retailer can reorder from his supplier. Our result can be treated a further step of Dada and $\mathrm{Hu}^{[14]}$ to explore the interfaces between operations and finance. To put it differently, our model can reduce to [14] when $\lambda=0$ and $M \rightarrow b$ (or $e=p$ ). This theorem shows that the bank's optimal interest rate $r^{*}$ is related to the bank's risk attitude, retailer's optimal order quantity $q^{*}$ and accordingly, the optimal interest rate depends on the double order policy. 
Corollary 5 The optimal interest rate increases in $M$.

Corollary 5 can be expected intuitively. A higher $M$ results in a higher primary order quantity, which suggests that the newsvendor need more capital and borrows more from the bank. Accordingly, the bankruptcy risk is higher. To hedging the risk, the bank would charge a higher interest rate.

In the context that follows, we extend the model to that the capital market is perfectly competitive ${ }^{[1]}$. Under this assumption, the bank is indifferent in financing the retailer and investing the risk-free project. Therefore, we have

$$
(w q-B) r-p \int_{0}^{x_{1}} F(x) \mathrm{d} x=(w q-B) r_{f} .
$$

Incorporating Equality (14) into the expected profit of the retailer, and maximizing the expected profit, we have the following theorem to reveal the optimal primary order quantity.

Proposition 2 Under perfectly competitive capital market assumption, the optimal primary order quantity is determined by the following equation

$$
e \bar{F}(q+M)+p\left(\bar{F}(q)-\bar{F}\left(x_{2}\right)\right)-w=0,
$$

which is the same as the case where there is no capital constraint.

This theorem shows that under perfectly competitive capital market assumption, the newsvendor acts as an un-capital-constrained one, which generalize the existing results ${ }^{[1,10]}$.

\section{Conclusions}

This study proposes a system consisting of a capital-constrained newsvendor-like retailer and a a risk-averse bank under Mean-Variance criterion. The retailer faces a random demand, which suggests that there exists bankruptcy risk for the retailer. To examine the joint effect of the operational and financial mechanism on mitigating the capital-constraint problem, we assume that the supplier offers a double-order policy with reorder quantity threshold and the bank offers an unlimited credit. Stackelberg equilibrium, i.e., the optimal primary order quantity and optimal interest rate, is derived mathematically. The analytical model shows that the reorder price and the reorder quantity threshold have significant impacts on the optimal decisions and bankruptcy risk. Extension considering a perfectly competitive capital market is given.

This papers aims to further explore the operations and finance interfaces, and derive some new insights. There are some interesting future directions worthy of attention. For example, the capital-constrained retailer has decisions involving both business and investment, therefore, how to apply loans and allocate the capital is an interesting problems. More risk-sharing contracts can be proposed to improve the capital-constrained system efficiency. More flexible operations can be considered to examine the impact on operations-finance decisions.

\section{References}

[1] Kouvelis P, Zhao W. Financing the newsvendor: Supplier vs. bank, and the structure of optimal trade credit contracts. Operations Research, 2012, 60(3): 566-580.

[2] Cai G G, Chen X, Xiao Z. The roles of bank and trade credits: Theoretical analysis and empirical evidence. Production and Operations Management, 2014, 23(4): 583-598.

[3] Ayyagari M, Beck T, Demirguc-Kunt A. Small and medium enterprises across the globe. Small Business Economics, 2007, 29(4): 415-434. 
[4] Sethi S P, Yan H, Zhang H. Quantity Flexibility contracts: Optimal decisions with information updates. Decision Sciences, 2004, 35(4): 691-712.

[5] Lau H S, Lau A H. Reordering strategies for a newsboy-type product. European Journal of Operational Research, 1997, 103(16): 557-572.

[6] Weng Z K. Coordinating order quantities between the manufacturer and the buyer: A generalized newsvendor model. European Journal of Operational Research, 2004, 156(1): 148-161.

[7] Xu X, Birge J R. Joint production and financing decisions: Modeling and analysis. Working Paper, Graduate School of Business, University of Chicago, 2004.

[8] Chao X, Chen J, Wang S. Dynamic inventory management with cash flow constraints. Naval Research Logistics, 2008, 55(8): 758-768.

[9] Buzacott J A, Zhang R Q. Inventory management with asset-based financing. Management Science, 2004, 50(9): 1274-1292.

[10] Jing B, Chen X, Cai G G. Equilibrium financing in a distribution channel with capital constraint. Production and Operations Management, 2012, 21(6): 1090-1101.

[11] Zhang B F, Wu D D, Liang L. Optimal option ordering and pricing decisions with capital constraint and default risk. IEEE Systems Journal, accepted and to be published, 2015, DOI: 10.1109/JSYST.2015.2460263.

[12] Zhang Q H, Zhao Q W. Research on loan-to-value ratio of inventory financing when demand is stochastic. Chinese Journal of Management Science, 2010, 5: 004.

[13] Ma Z H. The order quantity decision of newsboy retailer with financing. Journal of Systems Science and Information, 2014, 2(4): 358-365.

[14] Dada M, Hu Q. Financing newsvendor inventory. Operations Research Letters, 2008, 36(5): 569-573.

[15] Chen X, Wang A. Trade credit contract with limited liability in the supply chain with budget constraints. Annals of Operations Research, 2012, 196(1): 153-165.

[16] Chiu C H, Choi T M. Supply chain risk analysis with mean-variance models: A technical review. Annals of Operations Research, 2013: 1-19.

\section{Appendix}

Proof of Theorem 1 The first-order deviation of $x_{2}$ with respect to $q$ gives $\frac{\partial x_{2}}{\partial q}=1$.

Taking the first-order deviation of the expected profit of the retailer to w.r.t. $q$ results in

$$
\frac{\partial E \pi_{R}}{\partial q}=e-w-p\left(F(q+M)-\frac{\partial x_{2}}{\partial q} F\left(x_{2}\right)\right)+(p-e) F(q+M),
$$

which can be rewritten as

$$
\frac{\partial E \pi_{R}}{\partial q}=e \bar{F}(q+M)+p\left(\bar{F}(q)-\bar{F}\left(x_{2}\right)\right)-w .
$$

Taking the second-order deviation of the expected profit of the retailer w.r.t. $q$ derives $\frac{\partial^{2} E \pi_{R}}{\partial q^{2}}=$ $-e f(q+M)-p f(q)+p f\left(x_{2}\right)$.

Recalling $h(x)=\frac{f(x)}{\bar{F}(x)}, \frac{\partial^{2} E \pi_{R}}{\partial q^{2}}$ can be rewritten as $\frac{\partial^{2} E \pi_{R}}{\partial q^{2}}=-e h(q+M) \bar{F}(q+M)-$ $p h(q) \bar{F}(q)+p h\left(x_{2}\right) \bar{F}\left(x_{2}\right)$.

Due to $\bar{F}(q)>\bar{F}\left(x_{2}\right)>\bar{F}(q+M)$ and the IFR property of the demand distribution, it is easy to obtain that $\left.\frac{\partial^{2} E \pi_{R}}{\partial q^{2}}\right|_{\frac{\partial E \pi_{R}}{\partial q}=0}<0$, which is sufficient to prove the uniqueness of the optimal ordering level.

Therefore, the optimal order quantity satisfies first-order optimality condition, i.e., $e \bar{F}(q+$ $M)+p\left(\bar{F}(q)-\bar{F}\left(x_{2}\right)\right)-w=0$.

Proof of Theorem 2 Taking the first-order deviation of $x_{1}$ and $x_{2}$ with respect to $q$ derives $\frac{\partial x_{1}}{\partial q}=\frac{w(1+r)}{p}$ and $\frac{\partial x_{2}}{\partial q}=1$, respectively. 
Taking the first-order deviation of the expected profit of the retailer to the order quantity gives

$\frac{\partial E \pi_{R}}{\partial q}=e-w(1+r)-p\left(F(q)-\frac{\partial x_{1}}{\partial q} F\left(x_{1}\right)\right)-p\left(F(q+M)-\frac{\partial x_{2}}{\partial q} F\left(x_{2}\right)\right)+(p-e) F(q+M)$.

By replacing $\frac{\partial x_{1}}{\partial q}$ and $\frac{\partial x_{2}}{\partial q}$, the above formula is rewritten as

$$
\frac{\partial E \pi_{R}}{\partial q}=w(1+r) F\left(x_{1}\right)-p F(q)+p F\left(x_{2}\right)-e F(q+M)-w(1+r)+e .
$$

Let $\frac{\partial E \pi_{R}}{\partial q}=0$, we have $e \bar{F}(q+M)-w(1+r) \bar{F}\left(x_{1}\right)+p \bar{F}(q)-p \bar{F}\left(x_{2}\right)=0$.

Taking the second-order deviation of the expected profit of the retailer w.r.t. $q$ results in

$$
\frac{\partial^{2} E \pi_{R}}{\partial q^{2}}=\frac{(w(1+r))^{2}}{p} f\left(x_{1}\right)-p f(q)+p f\left(x_{2}\right)-e f(q+M)
$$

Recalling $h(x)=\frac{f(x)}{\bar{F}(x)}, \frac{\partial^{2} E \pi_{R}}{\partial q^{2}}$ can be rewritten as

$$
\frac{\partial^{2} E \pi_{R}}{\partial q^{2}}=\frac{(w(1+r))^{2}}{p} h\left(x_{1}\right) \bar{F}\left(x_{1}\right)-p h(q) \bar{F}(q)+p h\left(x_{2}\right) \bar{F}\left(x_{2}\right)-e h(q+M) \bar{F}(q+M) .
$$

Reminding $\bar{F}\left(x_{1}\right)>\bar{F}(q)>\bar{F}\left(x_{2}\right)>\bar{F}(q+M)$ and the IFR property of the demand distribution, it is easy to obtain that $\left.\frac{\partial^{2} E \pi_{R}}{\partial q^{2}}\right|_{\frac{\partial E \pi_{R}}{\partial q}=0}<0$, which is sufficient to prove the uniqueness of the optimal ordering level. For simplicity, let $K=\left.\frac{\partial^{2} E \pi_{R}}{\partial q^{2}}\right|_{\frac{\partial E \pi_{R}}{\partial q}=0}$ and $K<0$.

Proof of Corollary 1 Based on the implicit function derivative rule, we have

$$
\frac{\partial q^{*}}{\partial w}=\frac{(1+r) \bar{F}\left(x_{1}\right)-\frac{w q^{*}(1+r)^{2}}{p} f\left(x_{1}\right)}{K} .
$$

By reminding the proof of Proposition 1, we get the denominator is negative, then we discuss the numerator. Further,

$$
\begin{aligned}
& (1+r) \bar{F}\left(x_{1}\right)-\frac{w q^{*}(1+r)^{2}}{p} f\left(x_{1}\right) \\
= & (1+r) \bar{F}\left(x_{1}\right)-\frac{w q^{*}(1+r)^{2}}{p} h\left(x_{1}\right) \bar{F}\left(x_{1}\right) \\
= & (1+r) \bar{F}\left(x_{1}\right)\left(1-\frac{w q^{*}(1+r)}{p} h\left(x_{1}\right)\right) \\
> & (1+r) \bar{F}\left(x_{1}\right)\left(1-q^{*} h\left(q^{*}\right)\right) \\
= & (1+r) \bar{F}\left(x_{1}\right)\left(1-H\left(q^{*}\right)\right) .
\end{aligned}
$$

Due to $H\left(q^{*}\right)<1$, we can show the numerator is positive. Accordingly, we can conclude that $\frac{\partial q^{*}}{\partial w}<0$. Therefore, the optimal order quantity is decreasing in the wholesale price.

Proof of Corollary 2 Based on the implicit function derivative rule, we have

$$
\frac{\partial q^{*}}{\partial e}=\frac{M f\left(x_{2}\right)+\bar{F}\left(q^{*}+M\right)}{K} .
$$

It is straightforward to get that $\frac{\partial q^{*}}{\partial e}>0$. Therefore, the optimal order quantity is increasing in the re-order price, $e$. 
Proof of Corollary 4 Taking the first-order derivation of optimal order quantity w.r.t. $r$ results in $\frac{\partial q^{*}}{\partial r}=\frac{w \bar{F}\left(x_{1}\right)-\frac{w(1+r)\left(w q^{*}-B\right)}{p} f\left(x_{1}\right)}{K}$, where $K<0$. By reminding the proof of Proposition 1 , we get the denominator is negative, then we discuss the numerator, find it is positive as $w \bar{F}\left(x_{1}\right)-\frac{w(1+r)\left(w q^{*}-B\right)}{p} f\left(x_{1}\right)=w \bar{F}\left(x_{1}\right)-w x_{1} f\left(x_{1}\right)=w \bar{F}\left(x_{1}\right)\left(1-x_{1} h\left(x_{1}\right)\right)$.

It is easy to verify that the numerator is positive. Consequently, we can easily get $\frac{\partial q^{*}}{\partial r}<0$. Therefore, the optimal order quantity is decreasing in the interest rate, $r$.

Proof of Theorem 3 The first-order derivation of the utility function of the bank to her interest rate is given as $\frac{\partial U}{\partial r}=-p F\left(x_{1}\right) \frac{\partial x_{1}}{\partial r}+(w q-B)+w r \frac{\partial q}{\partial r}-\lambda\left(2(w q-B) w r^{2}+(w q-B)^{2} r-\right.$ $2 p x_{1} F\left(x_{1}\right) \frac{\partial x_{1}}{\partial r}+2 p w(1+r) \int_{0}^{x_{1}} F(x) \mathrm{d} x \frac{\partial q}{\partial r}+2 p(w q-B) \int_{0}^{x_{1}} F(x) \mathrm{d} x+2 p(w q-B)(1+r) F\left(x_{1}\right) \frac{\partial x_{1}}{\partial r}-$ $\left.2 p^{2} F\left(x_{1}\right) \int_{0}^{x_{1}} F(x) \mathrm{d} x \frac{\partial x_{1}}{\partial r}\right)$.

The derivative of bankruptcy threshold to the interest is given as $\frac{\partial x_{1}}{\partial r}=\frac{w(1+r)}{p} \frac{\partial q}{\partial r}-\frac{w q-B}{p}$. By replacing $\frac{\partial x_{1}}{\partial r}$, the above formula can be rewritten and simplified as $(w r(1-2 \lambda(w q-B) r)-$ $\left.w(1+r) F\left(x_{1}\right)(1-\lambda N)\right) \frac{\partial q}{\partial r}-(w q-B)\left(\bar{F}\left(x_{1}\right)-\lambda\left(2(w q-B) r+2 p \int_{0}^{x_{1}} F(x) \mathrm{d} x-N F\left(x_{1}\right)\right)=0\right.$, where $N=(2 p-1) x_{1}+2 p-2 p \int_{0}^{x_{1}} F(x) \mathrm{d} x$, and $\frac{\partial q}{\partial r}=\frac{w F\left(x_{1}\right) 1-x_{1} h\left(x_{1}\right)}{K}$.

Let $\frac{\partial E \pi_{B}}{\partial r}=0$, and we can get the condition that the optimal interest rate satisfies. 